\title{
LA-UR-12-25007
}

Approved for public release; distribution is unlimited.

Title:

Author(s):

Intended for:

\section{A Statistical Methodology for Quantifying Improvement in Predictive Capability: Key Features and Benefits}

Sigeti, David E.

Pelak, Robert A.

arXiv

Report

Disclaimer:

Los Alamos National Laboratory, an affirmative action/equal opportunity employer,is operated by the Los Alamos National

Security, LLC for the National NuclearSecurity Administration of the U.S. Department of Energy under contract DE-AC52-06NA25396. By approving this article, the publisher recognizes that the U.S. Government retains nonexclusive, royalty-free license to publish or reproduce the published form of this contribution, or to allow others to do so, for U.S. Government purposes.

Los Alamos National Laboratory requests that the publisher identify this article as work performed under the auspices of the

U.S. Departmentof Energy. Los Alamos National Laboratory strongly supports academic freedom and a researcher's right to publish; as an institution, however, the Laboratory does not endorse the viewpoint of a publication or guarantee its technical correctness. 


\title{
A Statistical Methodology for Quantifying Improvement in Predictive Capability: Key Features and Benefits
}

\author{
David E. Sigeti and Robert A. Pelak
}

September 11, 2012

In References [1, 2, 3], we proposed a statistical methodology for quantifying improvement in predictive capability. In this note, we summarize the key features and benefits of our approach.

We begin with a brief summary of our methodology. Broadly speaking, our approach models the predictive process statistically at a high level. Accordingly, we assume the existence of a body of experiments, the results of which have been (or will be) predicted. We can then treat the discrepancy between a prediction and the corresponding experimental result as a datum and the collection of such data informs us about the statistics of the prediction process. Specifically, we assume:

- The existence of a "legacy" simulation code that has been used to predict experiments in the past;

- The existence of a "new" simulation code that may be used to "postdict" (without calibrating to the known results) the experiments for which the legacy code originally produced predictions ${ }^{1}$;

- That it is possible to obtain predictions for the same experiments from both codes; and

- That it is possible to determine, in a given individual experiment, whether or not the new code has done a better job of predicting the

\footnotetext{
${ }^{1}$ In reality, we are really speaking here not just of legacy and new codes but of legacy and new modeling methodologies that include the codes. In order to avoid confusion with our use of the term "methodology" to refer to our statistical approach, we will use the term "code" to stand for the two modeling methodologies.
} 
experimental result than the legacy code. The paradigmatic example here is a single code output for which the quality of a prediction is determined simply by the absolute value of the discrepancy between the predicted and measured values, with a smaller absolute discrepancy being better.

We then point out that these assumptions allow us to reduce the information about legacy and new predictions of a given experiment to a single binary number, which takes the value of 1 if the new code did a better job at predicting the experiment and the value 0 otherwise. Then, assuming that we have a sample of paired predictions from the two codes and corresponding experimental results, we show how one may use Bayesian inference to obtain information about the probability, $\theta$, that, in an experiment chosen at random, the new code will provide a better prediction than the old. Of course, a value of $\theta$ greater than $1 / 2$ corresponds to an improvement in predictive capability. In particular, we show how to estimate $\theta$ based on observed predictions from the legacy and new codes and how it is possible to determine, in advance, how many new simulations will need to be run and compared to experimental results in order to have a given expected confidence in any detection of improvement or lack of same.

We envision our approach as one metric in a suite of tools and approaches for quantifying improvement in predictive capability. As such, it is appealing in that it:

\section{Provides a simple metric for comparing predictive capability across a suite of experiments;}

The principal quantity that is estimated in our approach, $\theta$, is a simple, intuitive measure of improvement in predictive capability - if $\theta$ is greater than $1 / 2$, then the new code will, more often than not, produce better predictions than the old.

\section{Makes minimal statistical assumptions;}

In our approach, it is not necessary to explicitly model the joint distribution of legacy and new prediction errors, nor their individual onevariable distributions, nor their correlations. The analysis is about as free of statistical modeling assumptions as one could possibly imagine.

\section{Evaluates codes based only on the quality of their predictions (black-box approach);}

Our approach does not require detailed knowledge of the codes and 
modeling that were used to make the predictions, knowledge that may very well not be available when one is working with legacy predictions. In particular, we do not need to know how many free parameters were used in the modeling. All that is required is that the predictions be genuine predictions, in that the models must not have been fit to the experimental results (of the specific experiments that were predicted).

\section{Uses pairing information when legacy and new codes are used to predict the same experiments;}

It is well known in statistics that, when comparing different classes of data such as predictions from legacy and new codes, statistical inference is much more powerful if the data from the different classes can be paired, provided of course that the statistical approach uses this pairing information. (See, for example, the sample problem in Box et al [4, Section 3.2]). Our approach uses the pairing information that is implicit in performing predictions of the same experiments using different codes. Note that a simple comparison of $\chi^{2}$ statistics for the legacy and new predictions effectively ignores the pairing information.

\section{Automatically takes account of "statistical significance";}

Because we do a Bayesian analysis, the fundamental statistical quantity of interest is the posterior distribution for $\theta$. The posterior distribution becomes more sharply peaked as more data is included in the analysis, guaranteeing that our confidence in our results increases appropriately as we include more data. Thus, our use of the posterior distribution automatically takes account of "statistical significance." See Figure 1 in Sigeti and Pelak [2] and the accompanying discussion in the text for an example.

6. Makes it possible to assess how many new simulations will need to be done;

In statistics, the term power analysis refers to the process of determining, in advance, how much data will need to be taken in order to provide a given level of confidence in the results of a statistical analysis of the data. Power analysis is used in the planning and the design of experiments, where it makes it possible to estimate the likely benefit to be obtained from a given amount of experimental effort [5].

When comparing the predictive capabilities of different simulation codes, and when prediction is expensive (in computer resources, staff time, 
etc.), it is desirable to know in advance how many predictions will need to be done in order to be able to make a statistically significant comparison. In other words, a power analysis is called for. Our approach provides such a power analysis. Indeed, the bulk of the material in our papers is devoted to power analysis.

In addition, we have generalized our original analysis to include $s e$ quential power analysis [3], so that, for example, a small number of comparisons may be used to inform decisions about how many additional comparisons will need to be done.

It is worth noting that power analysis is notoriously complex and the more complex the statistical modeling, the more complex the power analysis. The model that we use, the well-known binomial model, is pretty much the simplest statistical model possible and this simplicity makes a pretty-much complete power analysis possible.

\section{Does not require detailed modeling of experimental uncer- tainties;}

Modeling experimental uncertainties for legacy experiments is often extremely difficult. Often, experimentalists have not provided any experimental uncertainties at all. When they have, it is often difficult to interpret the quantities they have provided. For example, it may be unclear whether the stated uncertainties were intended as one-sigma or as two-sigma uncertainties, or as some kind of strict bounds. If the experiments were performed many years before the statistical analysis, there may be no way to answer such questions as the experimentalists may be unavailable due to career changes, retirement, or death. Even when the experimentalists are available, memories may be faulty or may fail altogether.

The interpretation of experimental uncertainties becomes even more difficult when one is analyzing multiple experiments performed over a long period of time by different experimentalists, or even different generations of experimentalists. Uncertainties in outputs from different experiments may have been assessed with very different methodologies everything from formal statistical analysis to expert judgment (which may itself derive from anything from long personal experience to folk knowledge) to a "scientific wild-assed guess" is likely to show up.

In light of these difficulties (which can show up in new as well as legacy experiments), it is good to have a statistical methodology available that 
is not dependent on detailed modeling of the experimental uncertainties. Our approach requires no such modeling.

One may reasonably ask, then, whether experimental uncertainty plays any role in our approach at all. It does, but (at present) only implicitly. In effect, the experimental uncertainty is aggregated with the modeling error in the codes to produce an overall assessment of predictions. If experimental error becomes large compared to the differences between legacy and new predictions, then the probability that a new prediction will be better than a legacy prediction will become more random, that is, $\theta$ will move toward $1 / 2$.

It would certainly be possible to incorporate knowledge about experimental uncertainties into our analysis. On the simplest level, it makes sense to take a quick look at predictions from legacy and new codes and to ask if they are generally within experimental uncertainty (assuming that one has at least a rough idea of what the experimental uncertainty should be). We think that this should be done in any case.

If predictions from both codes are generally within experimental uncertainties but one code does better than the other in a large percentage of the cases, then one should probably reexamine one's assumptions about experimental uncertainties, just as one would if a linear fit to some data produced a line that fit the data much better than the assumed experimental uncertainties should allow. If it is consistently possible to predict results to within a small fraction of experimental uncertainty, one's assumed uncertainty is probably too high.

More formally, it would be possible to separate the discrepancy between the prediction and the experimental value into a part due to experimental error and a part due to modeling error. Such an approach would require strong assumptions about the statistics of the predictions and of the experimental results and would lose the simplicity of the binomial model. Extending our analysis in this direction is not currently one of our priorities but we would be open to doing so if potential customers believe that it would be valuable.

At this point, it is appropriate to mention one important limitation of our current approach - it takes no account of how much better one code does than the other in predicting a given experiment. Rather, the quantitative difference in the discrepancies (between the two predictions and the experimental result) is reduced to a binary datum indicating which prediction was 
better.

Taking into account the full difference would provide a different look at the data that would, in all likelihood, provide us with better understanding than the simple binomial analysis alone. Of course, such an analysis would require much stronger assumptions on the statistics of the data (the discrepancies in particular), so we believe that it is best thought of as supplementing, rather than replacing, the simple binomial analysis that we have already done. Work on such an approach is currently underway.

Finally, we think that we should say a few words about the difference between comparing the quality of fits to data, on the one hand, and comparing predictive capabilities of different codes, on the other. When comparing quality of fits, one is asking how well different models (for example, different simulation codes) can be made to fit known data. In this context, questions of overfitting become very important because a model with a larger number of fitted parameters can often be made to fit data better than a model with a smaller number of fitted parameters, even if the second model is physically superior. Concern about overfitting leads to many complex and subtle questions and the use of techniques like information criteria (Akaike, Schwarz, or others) or Bayes factors when comparing qualities of fits. All these techniques depend critically on detailed modeling of experimental uncertainties and on detailed knowledge of the free parameters in the codes.

In the case of comparing predictive capabilities, on the other hand, it is much more a matter of the proof of the pudding being in the eating, provided one has a reasonable number of predictions to compare to experimental results. If a model has been shown to work well for prediction, we have strong evidence that it has not been overfit. In this case, we can proceed, as in our statistical methodology, to evaluate predictions without detailed modeling of either experimental uncertainties or the internals of the codes.

\section{References}

[1] David E. Sigeti and Robert A. Pelak. Using prediction ratios for power analysis. Technical Report LA-UR-11-05630, Los Alamos National Laboratory, 2011.

[2] David E. Sigeti and Robert A. Pelak. Are we getting any better? A methodology for quantifying improvement in predictive capability (U). In Proceedings of NEDPC 2011. Los Alamos National Laboratory, 2011. LA-UR-11-06952. 
[3] David E. Sigeti and Robert A. Pelak. Using a simple binomial model to assess improvement in predictive capability: Sequential bayesian inference, hypothesis testing, and power analysis. Technical Report LA-UR12-24643, Los Alamos National Laboratory, 2012.

[4] George E. P. Box, J. Stuart Hunter, and William G. Hunter. Statistics for Experimenters: Design, Innovation, and Discovery. Wiley-Interscience, second edition, 2005.

[5] Jacob Cohen. Statistical Power Analysis for the Behavioral Sciences. Routledge Academic, second edition, 1988. 